\title{
4D imaging of clay reveals soil carbon dynamics
}

\author{
JUDY YANG ${ }^{1}$, IAN BOURG ${ }^{1}$, XINNING ZHANG ${ }^{1}$, \\ HOWARD STONE ${ }^{1 *}$
}

${ }^{1}$ PrinCETON University, Princeton, NJ 08544, USA

(qingjuny@princeton.edu, bourg@princeton.edu, xinningz@princeton.edu, *correspondence: hastone@princeton.edu)

The amount of carbon in soils correlates with the abundance of certain clay minerals, an observation widely viewed as indicating that sorption of organic matter to clay surfaces confers some form of "protection" from microbial decomposition. Recent field and laboratory experiments, however, observed a decrease of clay-protected carbon, yet the mechanism is still unknown. The persistent uncertainties regarding the mechanisms and dynamics of carbon protection by clay minerals in the presence of microorganisms and exoenzymes hinder accurate predictions of soil carbon and future climate. Here we reveal previously unseen and unrecognized 4D observations (three spatial dimensions plus time) of dynamic carbon sorption and release processes inside transparent clay aggregates. We show that high-molecular-weight sugars $(>1 \mathrm{kDa})$ are physically separated within smectite clay aggregates while bacteria are confined to the periphery of the aggregates. In short, our results provide direct visual evidence of the role of sorption in protecting soil carbon from microbial decomposition. Furthermore, we demonstrate that upon addition of extracellular sugardegrading enzymes, irreversibly-sorbed highmolecular-weight sugars are readily broken down into smaller fragments that are rapidly release from the clay aggregates. Our results show that microbial activity and exoenzyme production can directly impact the efficacy of carbon-mineral-protection, with potentially vast impacts on soil carbon dynamics, whereas current global carbon models tend to represent microbial carbon dynamics and mineral protection as distinct, indirectly coupled phenomena. 